\title{
Vorwort zum zweiten Teil
}

Die naturphilosophische Fundierung der personalen Lebenssphäre ergab zwei Richtungen, in deren Überkreuzung sich die Personalität der Lebensführung als wirklich möglich abzeichnete. In der vertikalen Richtung des Vergleichs der personalen mit non-personalen Lebensformen wurde sie durch die Exzentrierung der Konzentrik in der Verhaltensbildung möglich. Die Person wurde als das exzentrische Dritte eingeführt, das ein doppeltes Verhältnis zu seinem Organismus gewinnen kann. Insofern die Person ihren Organismus als Instrument und Medium verwendet, hat sie ihn als Körper (Körper-Haben). Insoweit sie mit ihm in ihrem lebendigen Vollzug zusammenfällt, ist sie dieser Leib (Leib-Sein). In der horizontalen Richtung des Vergleichs verschiedener personaler Lebensformen ergab sich als dessen wirkliche Ermöglichung die Mitwelt. In ihr steht jede Person da, wo die andere Person steht. Sie erkennt ihre Stellung in der Stellung der anderen Person auf dem Hintergrund eines gemeinsam geteilten Geistes. Gleichwohl kann Personalität nicht in diesen inter-personalen Relationen des Geistes aufgehen, insofern jede Person in ihrem leiblichen Vollzug für sie selbst, aber auch für andere Personen nicht austauschbar, unvertretbar und unersetzlich ist. Wir haben es also - sowohl vertikal als auch horizontal gesehen - mit einem Strukturbruch zu tun, der die betroffenen Personen in die Dynamik von Verschränkungsaufgaben stellt, deren allgemeine Verwirklichungsrichtungen in den anthropologischen Grundgesetzen formuliert wurden.

In dem naturphilosophischen Rahmen wurde deutlich, dass es ohne diese Überkreuzung von mitweltlichen Relationen zwischen Personen und deren Körper-Leib-Differenzen personales Leben nicht geben kann. Damit ist zwar vieles ausgeschlossen (z. B. allein anorganische Materie oder reine Geister), aber auch unter den inter-personalen Relationen im Kontext von Körper-LeibDifferenzen noch vieles offen. Plessner verfährt in seiner Sozial- und Kulturphilosophie analog zu seinem Prozedere in der Naturphilosophie. Letztere hat die Aufgabe, die Ermöglichungsbedingungen für lebensweltlich qualitative Naturerfahrungen freizulegen, die auch Experten als Laien in ihrer personalen Lebensführung und künftigen Forschungspraxis in Anspruch nehmen. In der Sozial- und Kulturphilosophie handelt es sich um die Rekonstruktion derjenigen lebensweltlichen Strukturen, die der Qualität nach spezifisch soziokulturelle Erfahrungen in der Moderne ermöglichen, also auch von den modernen Sozial- und Kulturwissenschaften dafür in Anspruch genommen werden, dass sich ihre geistigen Unternehmungen in Zukunft lebensweltlich fortsetzen lassen. Die sozial- und kulturphilosophische Fundierung personaler Lebensformen stellt ebenso wenig eine bestimmte Sozial- und Kulturtheorie innerhalb der empiri- 
schen Sozial- und Kulturwissenschaften dar, wie durch die naturphilosophische Fundierung der Biowissenschaften eine bestimmte theoretische Biologie formuliert wird. Ähnlich wie Plessner seine naturphilosophische Aufgabe klar von der der theoretischen und empirischen Biologie zu unterscheiden wusste, da er selbst auch als Zoologe gearbeitet hat, bringt er auch seine Sozial- und Kulturphilosophie nicht mit der theoretischen und empirischen Sozial- und Kulturwissenschaft durcheinander, da er sich auch als Soziologe und Kulturwissenschaftler betätigt hat (zu den reflexiv fruchtbaren Bezügen der gegenwärtigen Soziologie auf Plessner siehe Lindemann 2014 u. 2018; Fitzi 2015; Rosa 2016).

Welche Ermöglichungsstrukturen nehmen nun aber sozial- und kulturwissenschaftliche Experten und lebensweltliche Laien in ihrer modernen Lebensführung dafür in Anspruch, der Qualität nach spezifisch soziokulturelle Phänomene erfahren, sie als solche verstehen und interpretieren zu können? Darauf antworte die soziokulturelle Wirklichkeit in der Moderne selber, so Plessner, indem in ihr eine Funktionalisierung von Rollen für Personen stattfinde. Die Funktionalisierung bedeute, dass die Erbringung bestimmter soziokultureller Leistungen in Gesellschaft und Kultur an die Ausübung einer bestimmten Rollenfunktion gebunden werde. Sie löse die Leistung als Funktionserfüllung in den soziokulturellen Verhältnisweisen ab von den privaten und gemeinschaftlichen Kontexten, in denen diese Leistung aus je persönlichen Beziehungen und Motiven heraus entstehe und in die sie lebensweltlich auch erneut einmünde. Moderne Gesellschaft und Kultur unterstellen insofern Funktionsteilung und Integration von Teilfunktionen. Mit der Funktionalisierung von Personenrollen generiert ein Bruch zwischen eher privat-persönlicher und eher gesellschaftlichöffentlicher Existenz, der die Person in ihre Aufgabe der Lebensführung stellt. Personalität lebt unter modernen Struktur- und Funktionsbedingungen in der Aufgabe, diese zwischen ihrer privaten und öffentlichen Differenz zu integrieren, was ihr nur in Grenzen wirklich möglich sein kann. An welche lebensweltlich-praktischen Voraussetzungen, Bedingungen und Folgen ist diese funktionale Abspaltung gebunden, wenn sie nicht selber dysfunktional werden und die künftig miteinander zu teilende Lebenswelt zerstören soll?

In der Funktion wird die Rolle als Rolle zwar abstrakt anschaulich und verständlich, weil von den konkreten empirischen Eigenheiten dieser oder jener Persönlichkeit ablösbar. Aber die wirkliche Explikation der Rolle, insofern sie nicht nur Funktion ist, setzt implizite und a-funktionale Praktiken voraus, aus denen die Rolle hervorgehen und in die sie auch erneut eingehen kann. Die funktionale Ablösung lässt sich nicht durch dualistische Abspaltungen beliebig steigern, bis am Ende die Körper-Leib-Differenzen und die interpersonalen Relationen ausgehebelt, also für die betroffenen personalen Lebewesen nicht mehr 
lebbar sind. Daher erschließt Plessner ein ganzes Spektrum von Ermöglichungspraktiken, die von der elementar-gemeinschaftlichen und repräsentativen Rolle über das öffentlich-private Doppelgängertum der Personen bis zu der erwähnten Funktionalisierung der Personenrollen reichen. Die Funktionalisierung von Rollen setzt deren lebensweltliche Kontexte voraus, da sie ohne letztere nicht allein aus sich reproduziert werden kann.

Daher reicht die Beobachtung von Funktionsleistungen nicht aus, die Untersuchung braucht den Rückgang in deren lebensweltliche Voraussetzungen und Folgen durch teilnehmende Beobachtung (Krüger 1990 u. 1993). Die teilnehmende Beobachtung an diesem Spektrum wäre keine teilnehmende mehr, wenn sie nicht die Potentiale des Spielens in und des Schauspielens mit den Personenrollen von den beteiligten Individuen her entfalten würde. Der Spielraum für die Individualisierung der Personalität liegt zunächst in diesem Spielen in und in diesem Schauspielen mit historisch bereits existierenden Rollen vor, in die jede/r/s hineingeboren wird. Diese Spiel-Potentiale werden ihrerseits geschützt, solange die Personen spielerisch mit ihren Verhaltensgrenzen, die sie in ihrem Lachen und Weinen erfahren, umgehen können, aber verletzt, insoweit ungespielt gelacht und geweint wird oder diese spezifischen Ausdrucksweisen für Grenzsituationen durch Gewalt verunmöglicht werden. Auch in Hinsicht auf weitere Phänomenbereiche im personalen Verhaltensspektrum wie die Leidenschaften und Süchte lässt sich zwischen ihrer Bedingtheit oder Unbedingtheit für die je individuell betroffenen Personen differenzieren. Inwiefern können letztere in Leidenschaften und Süchten ihre Erfüllung finden, indem sie sich diesen ganz hingeben und in ihnen aufgehen? Inwieweit besteht darin aber auch die reale Möglichkeit, dass sich nicht nur Allzumenschliches in außergewöhnlich Menschlichem zeigt, sondern auch in Inhumanes verkehren kann?

Das von Plessners Sozial- und Kulturphilosophie freigelegte Ermöglichungsspektrum wird in dem folgenden Teil zunächst unter dem Fokus der Individualisierung von Personalität anhand ihrer Rollen durchlaufen, so im 11. Kapitel, und sodann umgekehrt unter dem Fokus der Personalisierung des Individuums im Schauspiel von Rollen entfaltet, so im 12. Kapitel. Natürlich stellen diese Individualisierung und jene Personalisierung nicht zwei getrennte Bereiche in der lebensweltlichen Erfahrung der soziokulturellen Wirklichkeit dar. Es handelt sich phänomenal um zwei Richtungen, in denen die Individualisierung und die Personalisierung durch Rollenmedien hindurch miteinander verschränkt werden können. Indem Personalität im Rollenmedium individualisiert wird, wird auch Individualität in diesem Medium personalisiert (siehe Edinger 2017, 5. Kap.). Auf den Medienbegriff komme ich zurück (in Kap. 12.3.). 
Die dabei aufzuzeigenden Strukturbrüche und daraus erwachsenden Verschränkungsaufgaben in der personalen Lebensführung werden methodisch bewusst so beschrieben und verstanden, dass sie nicht mit einer bestimmten innerwestlichen Interpretation der Phänomene zusammenfallen müssen. Dieses Vorgehen trägt der philosophisch-anthropologischen Aufgabe Rechnung, dass durch sie innerwestliche als auch interkulturelle Vergleiche und deren Grenzen ermöglicht statt durch die Selbstbestätigungssucht der je eigenen Interpretation abgeschnitten werden. Es müssen nicht alle westlichen Strukturbrüche, umso weniger, wenn es für sie in außerwestlichen Soziokulturen Äquivalente gibt oder sich solche im weltgeschichtlichen Verkehr herausbilden, vorab im Namen von je eigener individueller und kollektiver Autonomie, von je eigener individueller und kollektiver Selbstbestimmung und Selbstverwirklichung zur Norm fixiert werden. Es könnte sich dabei um historisch kontingente Vor-Urteile allein der westlichen Moderne über die personale Lebenssphäre handeln, die sich im künftigen Austausch mit außerwestlichen Soziokulturen ändern lassen. Die Grenzen dieser einseitigen Interpretation, die immer auf das je eigene Selbst abhebt, werden immanent und exemplarisch deutlich, wenn man sich ernsthaft den existenziell bedeutsamen Themen des Liebens und Sterbens in den dann folgenden Kapiteln 13 und 14 zuwendet. Im 15. Kapitel, das diesen zweiten Teil beschließt, komme ich auf die Körper-Leib-Differenz zurück, die Personalität in die Integrationsaufgabe ihrer Lebensführung stellt. Ihre Untersuchung erfordert eine je nach Thema neue Kombination phänomenologischer, hermeneutischer und verhaltenskritischer Methoden, um theoretisch urteilsfähig zu werden.

Schon im ersten Teil des vorliegenden Buches wurde mit Plessner eine Alternative zu dem je auf das eigene Selbst zentrierten Zugang zur Naturphilosophie vorgeschlagen, weil er zum Verlust von Welt führt und daher nicht Lösung, sondern Teil der ökologischen Krise ist. Ich komme auf die Grenzen der westlichen Selbstinterpretation durch dualistische Abspaltungen zugunsten des Eigenen im dritten Teil des vorliegenden Buches zurück, wenn wir das vermeintlich westliche Ende der Weltgeschichte durch seine geschichtsphilosophische Fundierung erneut öffnen werden für nicht-westliche Modernen. Das Thema der Grenzen je selbstbezogener Interpretationen wird auch im IV. Teil, d. h. im Vergleich der Plessnerschen Philosophischen Anthropologie mit anderen Philosophien wieder aufgenommen werden. 